\title{
Histogram binwidth and kernel bandwidth selection for the spike-rate estimation Hideaki Shimazaki*1 $^{*}$ and Shigeru Shinomoto ${ }^{2}$
}

\author{
Address: 'Theoretical Neuroscience Group, RIKEN Brain Science Institute, Wako-shi, Saitama, Japan and ${ }^{2}$ Department of Physics, Kyoto University, \\ Kyoto, Japan \\ Email: Hideaki Shimazaki* - shimazaki@brain.riken.jp \\ * Corresponding author
}

from Eighteenth Annual Computational Neuroscience Meeting: CNS*2009

Berlin, Germany. 18-23 July 2009

Published: 13 July 2009

BMC Neuroscience 2009, I0(SuppI I):PII6 doi:I0.II86/I47|-2202-I0-SI-PII6

This abstract is available from: http://www.biomedcentral.com//47I-2202/I0/SI/PII6

(C) 2009 Shimazaki and Shinomoto; licensee BioMed Central Ltd.

Histogram and kernel methods have been used as standard tools for capturing the instantaneous rate of neuronal spike discharges in the neurophysiology community. These methods are left with one free parameter that determines the smoothness of the estimated rate, namely a binwidth or bandwidth. In most of the neurophysiology literature, however, the binwidth or bandwidth that critically determines the goodness of the fit of the estimated rate to the underlying rate has been selected by individual researchers in an unsystematic manner. Recently, we established a method for selecting the histogram binwidth [1] as well as the kernel bandwidth [2], with which the estimated rate best approximates the unknown underlying rate. The resolution of the optimized estimated rate increases, or the optimal bin/band-width decreases, with the number of spike sequences sampled. It is notable that the optimal bin/band-width diverges if only a small number of experimental trials are available from a moderately fluctuating rate process [3]. In this case, any attempt for characterizing the underlying spike rate will lead to spurious results. To assist those who are confronted with such paucity of data, we developed a method that can suggest how many more trials are needed until the set of data can be analyzed with the required resolution.

\section{References}

I. Shimazaki $\mathrm{H}$, Shinomoto S: A method for selecting the bin size of a time histogram. Neural Comput 2007, 19:1503-1527.

2. Shimazaki $H$, Shinomoto $\mathrm{S}$ : Kernel width optimization in the spike-rate estimation. In Neural Coding Montevideo Uruguay; 2007:120-123.
3. Koyama S, Shinomoto S: Histogram bin width selection for time-dependent Poisson processes. J Phys A: Math Gen 2004, 37:7255-7265. 